\title{
LAPAROSCOPIC RADICAL CYSTECTOMY - INITIAL EXPERIENCE
}

\author{
Deyan Anakievskii, ${ }^{1,2}$ Alexander Hinev ${ }^{1,2}$, Rostislav Marinov ${ }^{2}$, Inna Gocheva ${ }^{2}$, \\ Viktor Nikolov ${ }^{2}$ \\ ${ }^{1}$ Department of Surgery, Faculty of Medicine, Medical University of Varna \\ ${ }^{2}$ Clinic of Urology, St. Marina University Hospital
}

\begin{abstract}
INTRODUCTION: Radical cystectomy with extended pelvic lymph node dissection is the standard treatment for muscle-invasive bladder cancer. The aim of this study is to report our initial experience of our series of 22 patients who underwent laparoscopic radical cystectomy with different urinary diversion.

MATERIALS AND METHODS: Between March 2015 and March 2016, 22 patients have undergone laparoscopic radical cystectomy with different types of extracorporeal urinary diversion. Patients were aged 54 to 85 (average age 66.3) with different clinical stages of the disease. In all of the cases a transperitoneal laparoscopic radical cystectomy with five ports was performed with bilateral extended pelvic lymph node dissection.

RESULTS: In 5 cases we performed radical cystectomy with subsequent ureterocutaneostomy, in 7 cases ileal conduit according to the method of Bricker was performed, and in 10 cases we formed an orthotopic bladder from an ileal loop by the method of Hautmann. All operations were performed with an average blood loss of $270 \mathrm{ml}$, with an average operating time of 5 hours, and an average hospital stay of 7 days. No conversion was required in any of the cases. The patients were observed postoperatively. Early complications (within 30 days) occurred in 2 patients, and late complications occurred in 3 patients.

CONCLUSION: Laparoscopic radical cystectomy is possible, although technically difficult, with significant reduction in patient morbidity. With more experience and an improvement of the surgical technique, laparoscopic radical cystectomy with different types of derivation becomes an alternative surgical method for treating patients with localised muscle invasive bladder carcinoma.
\end{abstract}

Keywords: bladder cancer, laparoscopic radical cystectomy, orthotopic, conduit

Address for correspondence:

Deyan Anakievski, $M D$

St. Marina University Hospital

Clinic of Urology

1 Hristo Smirnenski Blvd

9010 Varna, Bulgaria

e-mail:dejan_anakievski@yahoo.com

Received: February 25, 2017

Accepted: March 20, 2017

\section{INTRODUCTION}

Bladder cancer accounts for 63,000 new cases and around 13,000 deaths annually in the USA, and 357,000 cases with 145,000 deaths worldwide annually. Surgery plays a major role in the treatment of all stages of invasive bladder cancer. Open radical cystectomy (ORC) with pelvic lymph node dissection (LND), is currently regarded as the gold standard treatment for treating muscle-invasive and non-mus- 
cle invasive high-grade bladder cancer (1). However, the procedure is associated with high morbidity including increased blood loss, prolonged hospital stay and slower recovery. The operative goals of surgery (open RC, ORC, or laparoscopic RC, LRC) are to ensure negative surgical margins and an adequate LND (2). Radical cystectomy (RC) with a pelvic lymphadenectomy provides excellent local control and longterm survival after muscle-invasive bladder cancer. Traditionally, RC is performed by an open surgical approach, however, recently there have been more and more reports for minimally invasive approaches when treating invasive bladder cancer and these approaches have been established in numerous centres across the world $(3,4)$. In fact, with the advancement of the laparoscopy equipment and the increased skills of urologists performing minimally invasive surgery, laparoscopic radical cystectomy (LRC) has become a viable alternative to open radical cystectomy (ORC).

We present our initial experience and mid-term outcomes of LRC with different types of urinary diversion.

\section{MATERIALS AND METHODS \\ Patient selection}

The selection of suitable patients for LRC is an

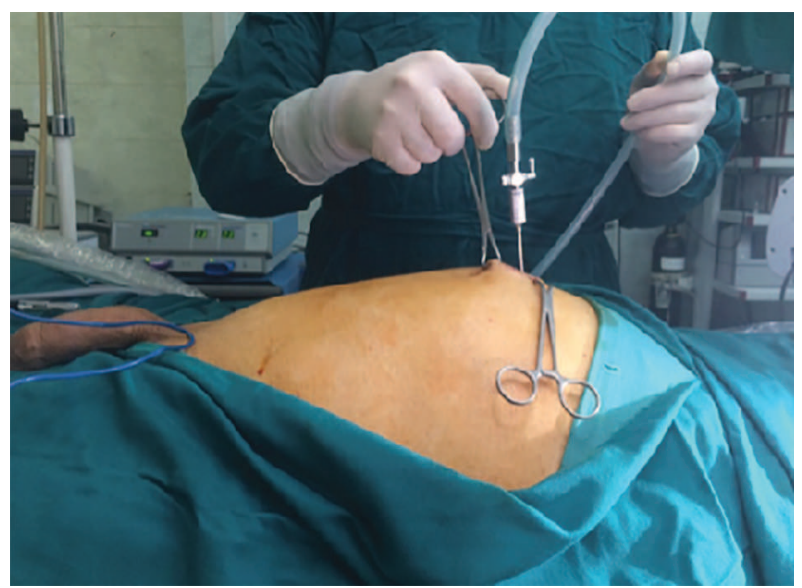

tion criteria should incorporate both patient and tumour characteristics. Uncontrolled bleeding diatheses, intra-abdominal infections and ascites remain general contraindications to laparoscopy, and probably to open surgery as well. Obesity is a relative contraindication, increasing the surgeon's fatigue and the operative complexity due to the absence of tissue planes and heavier manipulation of the abdominal wall. Obese patients are more easily handled with robotic-assisted laparoscopic surgery (5). A previous abdominal surgery also increases the surgical difficulty. Each patient's situation should be assessed, again taking into consideration the surgeon's experience. Older patients with cardiac or respiratory compromise have traditionally been excluded from laparoscopic surgery but recent reports showed low morbidity for such elderly patients (6).

\section{Patient Preparation:}

Laxative for colon cleaning and fasting for 8 hours were the preoperative preparations, also typing and blood reserve were routinely performed. Antibiotic prophylaxis was done to all patients prior to surgery. All patients were operated on under general anesthesia and insertions of urethral catheter and nasogastric tube were performed. Elastic bandage of both legs was placed for thromboembolic events.

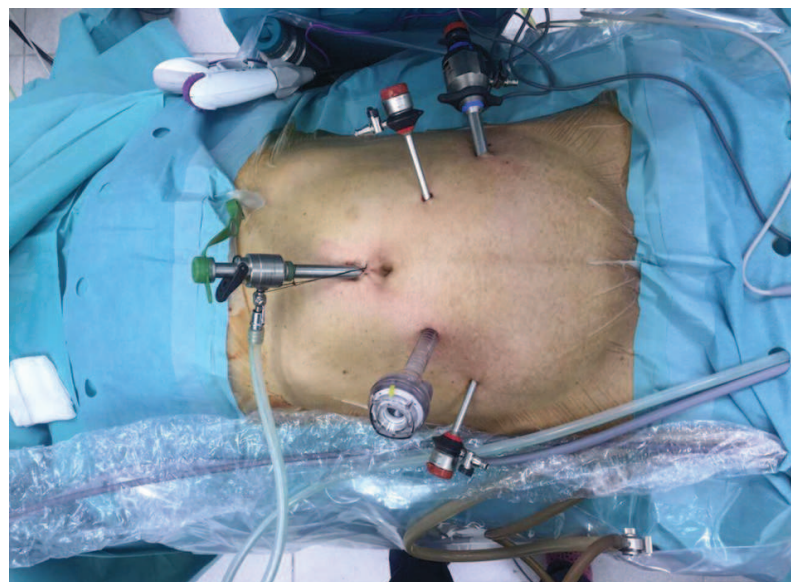

Fig. 1. Fan-shaped transperitoneal placement of 5 trocars for laparoscopic radical cystectomy

essential step to ensure satisfactory outcomes. The selection process should reflect the surgeon's experience, with easier cases (thin patients with early tumours and with no previous surgery or radiotherapy) attempted initially, and more complicated cases introduced in a progressive stepwise manner. Selec-
Pneumoperitoneum was created with a Veress needle, one $10 / 11 \mathrm{~mm}$ trocar was inserted above the umbilicus and a 0 degree lens was used for a review of the abdominal cavity. The other four trocars were inserted under optical control and were arranged in an 
Deyan Anakievski, Alexander Hinev, Rostislav Marinov et al.

inverted $\mathrm{V}$ shape with one trocar at the apex for the lens, two other of $10 / 12 \mathrm{~mm}$, and two final of $5 \mathrm{~mm}$ along the anterior-superior iliac spines (Fig. 1) (7).

For the operation we used different instruments like the LigaSure system and harmonic scalpel to dissect the tissue. For ligation of the vessels and other structures we used metal and plastic clips. After incision of the posterior plate of the peritoneum we identified the ureters, dissected distally to the drip into the bladder, ligated using a Hem-o-lok clip and cut. The distal margins of both ureters were sent for frozen section biopsy. After that we started with a dissection of the bladder base going down to the seminal vesicles, and the posterior surface of the prostate followed by a dissection of the Retzius space. After an incision of the fascia pelvica we dissected the prostate apex and went to the urethra, which was cut with a long stump. The extended lymph nodes dissection included obturator, external, internal and common iliac, presacral, paraaortic and paracaval lymph nodes. In females, the procedure was started from a dissection of the uterus ligaments and peritoneum in the Douglas cavity. The bladder, uterus, adnexa with anterior vaginal wall and lymph nodes were removed transvaginally.

The specimen was placed in an endobag after being removed through a $5-7 \mathrm{~cm}$, middle subumbli- cal incision. In women the final surgical specimens were removed vaginally, and the dome of the vagina was then sutured. Then the left ureter was transposed under the sigmoid mesocolon to the right side. The lymphadenectomy boundaries included the bladder medially, the genitofemoral nerve laterally, Cloquet's node distally, the obturator nerve and its vessels posteriorly, and the mid-common iliac vessels proximally.

We used three different types of urinary diversions according to the intraoperative frozen sections and the patient's condition: Hautmann orthotopic neobladder (Fig. 2), Bricker ileal conduit (Fig. 3 ), and cutaneous ureterostomy. The ileal conduit is a popular technique of urinary diversion after radical cystectomy. Ileal neobladder reconstruction was performed only in selected patients with usable urethra and patients with indication for neobladder. $\mathrm{Cu}-$ taneous ureterostomy was performed in patients at an advanced age and significant comorbidity (diabetes, anemia, COPD) (8). All urinary diversions were performed extracorporeally. In cases where an orthotopic urinary diversion was being performed, the neobladder is constructed extracorporeally, replaced inside the abdomen, and neobladder-urethral anastomosis is made laparoscopically as the final step of the procedure.
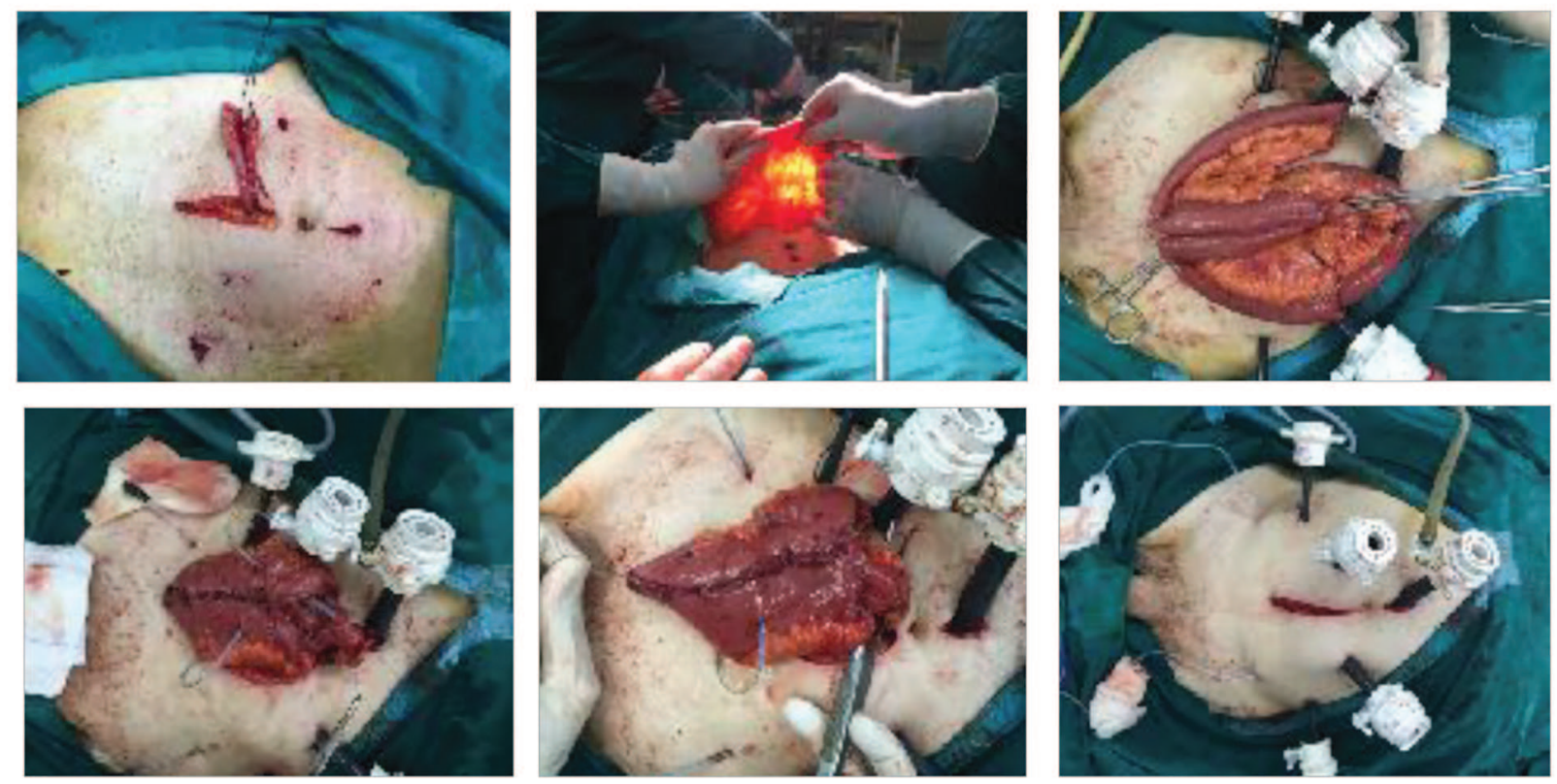

Fig. 2. Technique of making an orthotopic neobladder extracorporally 

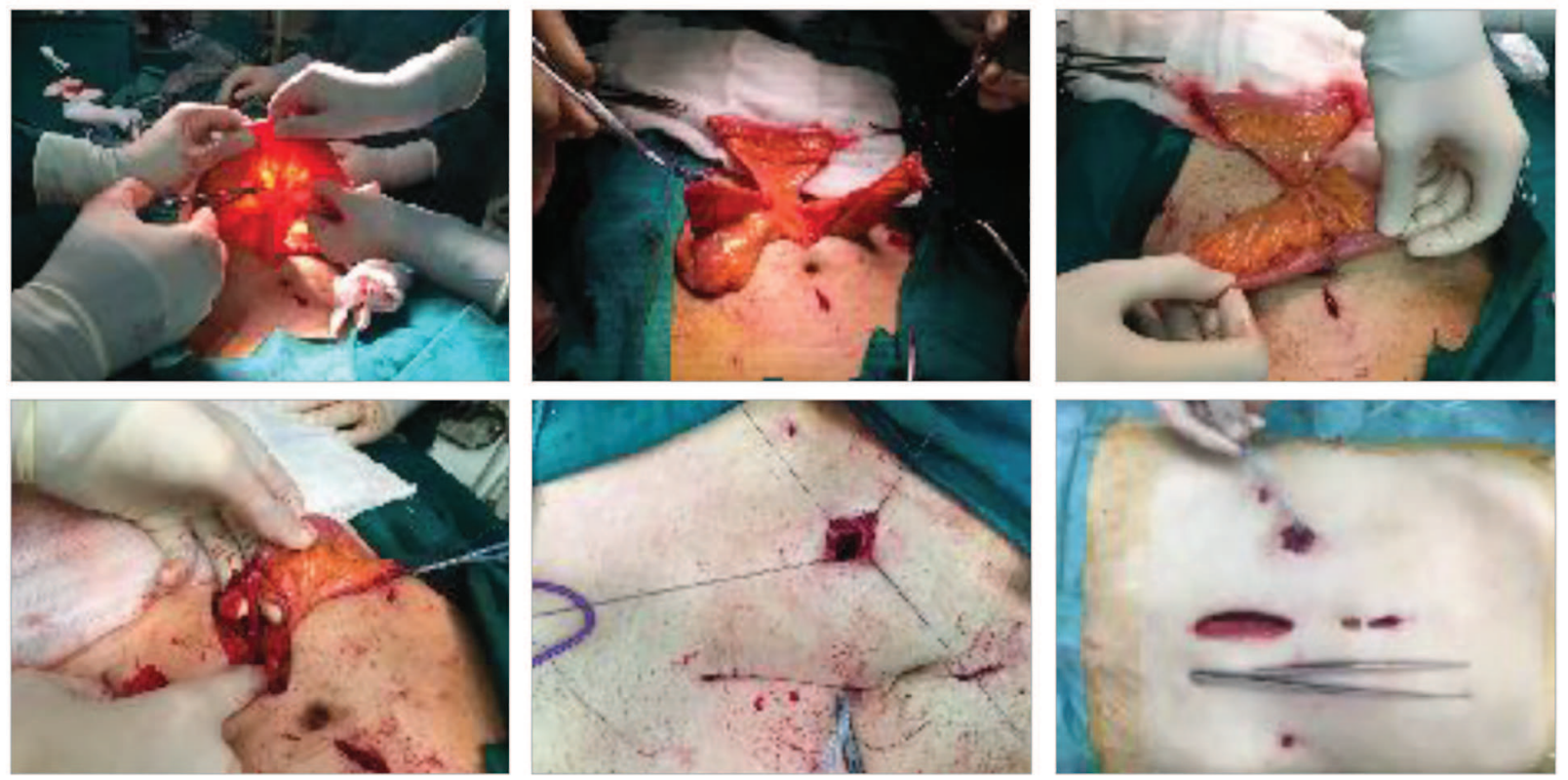

Fig. 3. Technique of making Bricker conduit extracorporally

In 5 cases (23\%), we performed radical cystectomy with subsequent ureterocutaneostomy, in 7 cases (32\%) ileal conduit according to the method of Bricker was performed, and in 10 cases (45\%) we formed an orthotopic bladder from an ileal loop by the method of Hautmann.

\section{Follow-up Methods:}

Postoperative follow-up during the first year was conducted at 3-month intervals, during the second year - at 6-month intervals, and annually thereafter. Follow-up included medical history, physical examination, and routine biochemical blood examination. Urography, ultrasonography of the abdomen, and chest X-rays were performed at 3, 6, and 12 months postoperatively, annually thereafter unless otherwise clinically indicated. Abdominal/pelvic computed tomography scans were performed 6 months postoperatively and then annually. The patients' complications were catalogued during hospitalisation and in clinical attendance for 90 days after surgery.

\section{RESULTS}

\section{Patients Characteristics:}

Perioperative and oncologic data were collected prospectively. Twenty-two patients, from which 18 men and 4 women, were operated on. The median age and body mass index were 66 years (range 50 to 85 ) and $22.65 \mathrm{~kg} / \mathrm{m}^{2}$ (range 18.14 to 30.08 ), respectively. Patients were stratified according to the American Society of Anesthesiologists classification: class 1 - 5 patients (23\%); class 2 - 12 patients (54\%); class 3 - 5 patients (23\%). Thirteen patients had a history of previous abdominal surgery, repair of bowel perforation, herniorrhaphy, laparoscopic cholecystectomy, and hysterectomy (Tabl. 1).

Radical cystectomy was completed laparoscopically in all patients without conversion to the open technique, and there was no perioperative mortality. The median operating time was 300 min (range 180 to 420 ). The median estimated blood loss (EBL) was $270 \mathrm{~mL}$ (range 150 to 500 ), with a transfusion rate of $3(13.3 \%)$. The mean number of lymph nodes removed with EPLND was 19.3 (range 6 to 36). The median range of postoperative pain was 3 days (2-6). The median time to oral intake was 5 days (range 4 to 7), and the median postoperative hospital stay was 7 days (range 5 to 12) (Tabl. 2). 
Deyan Anakievski, Alexander Hinev, Rostislav Marinov et al.

Table. 1. Patient preoperative characteristics

\section{Patient characteristics}

\begin{tabular}{|c|c|}
\hline \multicolumn{2}{|l|}{ Gender } \\
\hline Men, $\mathrm{n}$ & $18(82 \%)$ \\
\hline Women, $\mathrm{n}$ & $4(18 \%)$ \\
\hline Median age, yrs (range) & $66(50-85)$ \\
\hline Median BMI kg/m² (range) & $22,65(18,14-30,08)$ \\
\hline \multicolumn{2}{|l|}{ ASA score, n (\%) } \\
\hline 1 & $5(23 \%)$ \\
\hline 2 & $12(54 \%)$ \\
\hline 3 & $5(23 \%)$ \\
\hline \multicolumn{2}{|l|}{$\begin{array}{l}\text { Previous abdominal or pelvic } \\
\text { surgery, } n \text {. }\end{array}$} \\
\hline Repair of bowel perforation & 3 \\
\hline Herniorrhaphy & 4 \\
\hline Laparoscopic cholecystectomy & 3 \\
\hline Hysterectomy & 3 \\
\hline Smoking history, n (\%) & $20(91 \%)$ \\
\hline \multicolumn{2}{|l|}{$\begin{array}{l}\text { Preoperative clinical T stage, } \\
\text { n (\%) }\end{array}$} \\
\hline $\mathrm{cTa}$ & $1(4,5 \%)$ \\
\hline cT1 & $2(9 \%)$ \\
\hline cT2 & $15(68,5 \%)$ \\
\hline cT3 & $3(13,5 \%)$ \\
\hline cT4 & 0 \\
\hline Tis & $1(4,5 \%)$ \\
\hline \multicolumn{2}{|l|}{$\begin{array}{l}\text { Preoperative histological } \\
\text { grade, } \mathbf{n}(\%)\end{array}$} \\
\hline G I & 0 \\
\hline G II & $5(23 \%)$ \\
\hline G III & $17(77 \%)$ \\
\hline
\end{tabular}

\section{Complications:}

Complications were classified as immediate, early postoperative 30 days after the operation, and late postoperative after the 30th day. Early complications (within 30 days) occurred in 2 patients, in whom we observed paralytic ileus for 5 to 7 days, which was managed conservatively. Late complications occurred in 3 patients, consisting of stenosis of the ureteroileal anastomoses, which were managed with re-anastomosis two months after the surgery.

\section{DISCUSSION}

The first laparoscopic radical cystectomy (LRC) was performed by Parra et al. in the 90s (9), and in
Table. 2. Operative data

\section{Operative data}

Median (range) operating time,

$300(180-420)$

min.

Median (range) estimate blood loss, 270 (160-500)

ml.

Transfusion rate, $\mathrm{n}(\%)$

$3(13,5 \%)$

Conversion rate, $\mathbf{n}$

Extended lymph node dissection, $\mathbf{n}$

Mean (range) no. of lymph nodes

$19,3(6-36)$

removed, $\mathbf{n}$

\section{Urinary derivation}

UCS, n (\%)

$5(23 \%)$

Bricker, n (\%)

$7(32 \%)$

Orthotopic, n (\%)

$10(45 \%)$

Postoperative complications

Early (to 30 day), n (\%) $2(9 \%)$

Late (after 30 day), n (\%)

$3(13,5 \%)$

Median (range) hospital stay, days

$7(5-12)$

Median (range) postoperative pain, $3(2-6)$

days

1995 Sanchez de Badajoz et al. reported the first laparoscopic radical cystectomy (10). In 1995, combined laparoscopic and transvaginal anterior pelvic exenteration for bladder cancer was described by Puppo et al. in five cases (11). Completely intracorporeal LRC with a continent urinary diversion (rectal sigmoid pouch) in 5 cases was done in 2001 by Turk et al. (12). The first purely laparoscopic ileal conduit and the first purely laparoscopic orthotopic Studer neobladder reconstruction were performed in 2000 and 2002 by Gill et al. (13). At present there is no long-term oncologic data in large series after laparoscopic RC (LRC).

Contemporary cystectomy series with a 10 -year follow-up show that surgery cures the majority of patients with muscle-invasive tumours that are confined to the bladder (pathologic [P] stage pT2), about half of those with extravesical disease (stage pT3-4), and a significant minority with positive $(\mathrm{N})$ pelvic lymph nodes $(2,14)$. Patients who are likely to develop a recurrent disease are easily identified thanks to pathologic information provided by surgery. Unrec- 
ognized and untreated nodal or distant metastases present at the time of surgery lead to death of a large proportion of patients with bladder cancer.

Considerable debate during the initial experience with LRC focused on the urinary diversion technique and whether an intracorporeal technique is more beneficial than an extracorporeal technique (through mini-laparotomy). Although the intracorporeal technique is aesthetically superior, particularly in women, where a vaginal specimen extraction can be obtained, resulting in a truly 'incisionfree' cystectomy, the intracorporeal technique results in a significantly longer operation and has been associated with a higher rate of bowel-related complications in the initial series (15). Although intracorporeal bowel surgery is being reported more frequently, particularly with robotic assistance, most surgeons prefer to construct the urinary diversion through mini-laparotomy that is also used for extracting the surgical specimen.

The limit of dissection of lymph nodes is variable among surgeons and institutions. Nodal tissue is removed from the area surrounding the iliac vessels, between the genitofemoral nerve laterally, the node of Cloquet distally, and the obturator fossa medially. It is our preference to limit the dissection to the midpoint or the origin of the common iliac artery, but a successful extended lymphadenectomy can be done laparoscopically with a proximal extent as high as the origin of the inferior mesenteric artery from the distal aorta $(16,17,18)$. Presacral nodes are also removed, and this is facilitated by adequate mobilisation of the sigmoid colon.

In a prospective non-randomised comparison of the operative morbidity of 38 LRC and 30 ORC, blood loss, transfusion rate, minor complications, mortality, opioid requirement, resumption of oral intake and hospital discharge were all significantly shorter in the LRC group (19). A retrospective comparison of the oncological outcome of 36 LRC to 34 historical matched controls undergoing ORC showed no difference between the groups in terms of 3-year overall, cancer-specific or recurrence-free survival, even when the patients were divided into organ-confined (T1, T2) and extravesical (T3, T4) groups (20).

The largest published study currently available reported the oncological outcomes of 171 patients operated laparoscopically with a median follow-up of 3 years (21). All patients had an orthotopic ileal neobladder constructed extracorporeally. With impressive operative results of no open conversions or deaths, a median operating time of $325 \mathrm{~min}$, and a median blood loss of $270 \mathrm{~mL}$, a pathological evaluation showed no positive surgical margins, extravesical $(\mathrm{T} 3, \mathrm{~T} 4)$ disease in $34 \%$, and positive lymph nodes in $22 \%$. Local recurrence occurred in $5 \%$ of patients, while $14 \%$ had distant recurrence, and $1 \%$ had both local and distant recurrence. The 5-year overall, cancer-specific and recurrence-free survival rates were $74 \%, 81 \%$ and $73 \%$, respectively, with a median follow-up of 37 months, and only $32 \%$ of the patients completed 5 years of follow-up.

\section{CONCLUSION}

In experienced hands, LRC is an acceptable minimally invasive alternative to ORC in selected patients, with the main advantage of decreased blood loss and postoperative pain, as well as a shorter hospital stay and recovery. LRC has become established as a viable and less invasive option to ORC in selected patients. Improved cosmesis, decreased postoperative pain and shorter recovery continue to make the technique attractive to patients and surgeons alike. Careful patient selection and advanced laparoscopic skill are required for optimal outcomes. Continued developments in the field of robotics and single-site surgery promise significant improvements over the current techniques and outcomes.

RCs have coincided with the widespread adoption of laparoscopic techniques in urology, resulting in the development of LRC, which is rapidly becoming a viable treatment replacement for ORC. Significant worldwide experience has developed the technique from early single case reports to significantly more mature series with reproducible techniques $(15,21)$.

\section{REFERENCES}

1. O'Dea M, Furlow W. Nephropexy: fact or fiction? Urology 1976;8:9-12.

2. Hubner WA, Schramek P, Pfliiger H. Laparoscopic nephropexy. J Urol 1994;152:1184-1187.

3. Elashry OM, Nakada SY, McDougall EM, Clayman RV. Laparoscopic nephropexy: Washington University experience. J Urol 1995;154:1655-1659. 
4. Hahn E. Die operative Behandlung der beweglichen Niere durch Fixation. Zentralbl. Chir. 1881; 29: 449- 556.

5. Harrison LH, Glenn JF. Nephropexy. Urol. Surg. 1969; 3: 253-5.

6. Wandschneider G, Haas P, Leb G, Passath A. Indika- tionsstellung und Erfolgsbeurteilung der Nephropexie mit Hilfe der kombinierten Isotopenuntersuchung der Nieren. Urologe A 1972; 3: 161-9.

7. Wandschneider G. Results and problems of nephropexy. Urologe 1966; 5: 129-32.

8. Hoenig DM, Hemal AK, Shaihav AL, Clayman RV. Nephroptosis: A 'disparaged' condition revisited. Urol- ogy 1999; 54: 590-6.

9. Boeminghaus H. Urologie. Werk Verlag, MünchenGrafeling, 1971.

10. Fornara P, Doehn C, Jocham D. Laparoscopic neph- ropexy: 3-year experience. J. Urol. 1997; 158: 1679-83.

11. McDougall EM, Afane JS, Dunn MD, Collyer WC, Clayman RV. Laparoscopic nephropexy: Long-term follow-up. Washington University experience. J. Endourol. 2000; 14: 247-50.

12. Rassweiler JJ, Frede T, Recker F, Stock C, Seemann O, Alken P. Retroperitoneal laparoscopic nephropexy. Urol. Clin. North Am. 2001; 28: 137-44.

13. Harrison, LH. Nephropexy. In: Urologid Surgery, 3rd ed. Edited by J. F. Glenn. Philadelphia: J. B. Lippincott Co., pp. 253-355, 1983.

14. Hubner WA, Schramek P, Pfluger H. Laparoscopic nephropexy. J. Urol. 1994; 152: 1184-7.

15. Matsui $Y$, Matsuta $Y$, Okubo K, Yoshimura K. International Journal of Urology (2004) 11, 1-6 